\title{
Pseudoaneurysm of the superficial femoral artery detected by emergency medicine bedside ultrasound
}

\author{
Firat Bektas • Secgin Soyuncu
}

Received: 23 December 2009/Accepted: 19 April 2010/Published online: 21 August 2010

(C) Springer-Verlag London Ltd 2010

\begin{abstract}
Background Femoral artery pseudoaneurysm following by cardiac catheterization is a serious groin complication requiring careful assessment and prompt intervention.

Aims Risk of femoral artery pseudoaneurysm is estimated at 0.6 to 17 following diagnostic and interventional procedures.

Methods The clinical usage of bedside ultrasonography as part of the physical examination by attending emergency physicians has increased significantly over recent years.

Results Bedside Emergency Department ultrasonography provides the clinician with critical information noninvasively, rapidly determining various anatomical structures. Conclusions We presented the case of a femoral artery pseudoaneurysm detected by the bedside emergency department ultrasonography secondary to angiographic catheterization.
\end{abstract}

Keywords Pseudoaneurysm · Ultrasonography .

Emergency medicine

\section{Case report}

A 51-year-old woman presented to the emergency department (ED) with a 2-day history of right groin pain, discoloration

This study was supported by the Akdeniz University Research Foundation

F. Bektas $(\bowtie) \cdot$ S. Soyuncu

Department of Emergency Medicine,

Akdeniz University Faculty of Medicine,

07059 Antalya, Turkey

e-mail: fbektas@akdeniz.edu.tr and swelling of the inguinal area. Six days before presentation, diagnostic angiographic catheterization was performed in her right femoral area. On physical examination, the inguinal area was swollen, tender and ecchymotic. There were no pulsatile masses. Emergency department ultrasonography (US) performed at the bedside revealed a pulsating anechoic lesion communicating with the superficial femoral artery by using a $10-\mathrm{MHz}$ linear probe (Fig. 1). Doppler examination of the patient showed a $31 \times 26$ - $\mathrm{mm}$ pseudoaneurysm in direct continuity with the right femoral artery (Fig. 2a and b). She was surgically treated and discharged from the hospital fully recovered 10 days later.

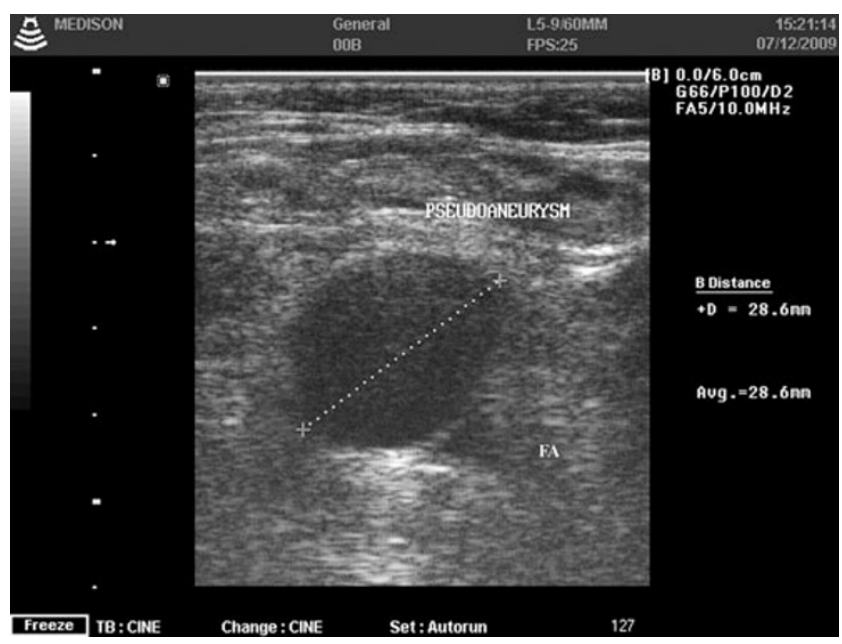

Fig. 1 Pseudoaneurysm of the superficial femoral artery. There is an anechoic lesion communicating with the right superficial femoral artery 

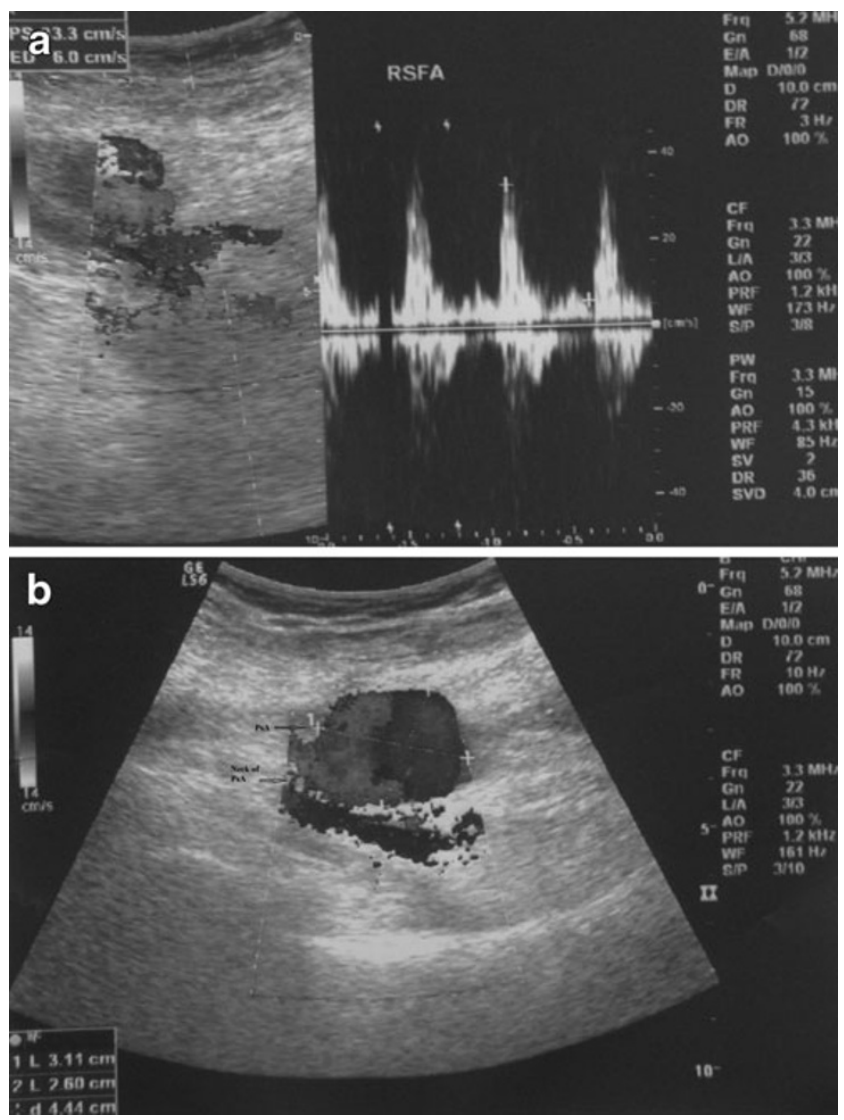

Fig. 2 a and b: Duplex Doppler US image depicts a $31 \times 26-\mathrm{mm}$ pseudoaneurysm $\left(P_{S A} A\right)$ with bidirectional flow within the neck

\section{Discussion}

A painful mass in the femoral area should heighten the clinician's index of suspicion for a possible pseudoaneurysm. Differential diagnoses of pseudoaneurysm include abscess and hematoma formation, cyst, malignant mesen- chymal tumor and lymphadenopathy, which may be the landmark of sexually transmitted infections such as lymphogranuloma venereum, gonorrhea or cancers such as lymphoma. Differential diagnosis should also include true femoral aneurysm.

The diagnosis of a femoral pseudoaneurysm may be performed with invasive and noninvasive methods: color Doppler US, CT angiography, magnetic resonance angiography and conventional angiography [1]. All of these conditions may be evaluated with bedside ED US. Using B-mode imaging, a pseudoaneurysm will usually appear as an anechoic lesion communicating with the femoral artery in the femoral area; this mode is useful for measuring pseudoaneurysm size and compression of adjacent structures [2]. Other advantages of ED US examination are that it is a simple, rapid, inexpensive and useful method for evaluating pseudoaneurysyms. Duplex ultrasound is the diagnostic test of choice and demonstrates a high velocity jet through a defect in the arterial wall on color flow imaging. It is, however, a technique that is operator dependent.

In conclusion, US examination may be a valuable tool in the ED to perform the differential diagnosis of a superficial femoral pseudoaneurysm.

\section{Conflict of interest None}

\section{References}

1. Saad NE, Saad WE, Davies MG, Waldman DL, Fultz PJ, Rubens DJ (2005) Pseudoaneurysms and the role of minimally invasive techniques in their management. Radiographics 25(Suppl 1):S173S189

2. Morgan R, Belli AM (2003) Current treatment methods for postcatheterization pseudoaneurysms. J Vasc Interv Radiol 14 (6):697-710 